cysteine proteases was examined. Fluorogenic substrates analysed include those for various inflammatory proteases including elastase-like (MeOSuc-AAPV-AMC), MMPs (MCA-PLGL-Dpa-ARNH2), trypsin-like (Z-GGR-AMC) and chymotrypsin-like (SucAAPF-AMC). Substrate hydrolysis by a relevant recombinant enzyme ( \pm inhibitors) was analysed as a control.

Results Data analysis indicates that alternative enzymes actively hydrolyse substrates designed to be specific for one group of proteases. Inhibitors specific for metallo, trypsin-like, chymotrypsinlike and cysteine proteases all decreased elastase-like substrate turnover $(\sim 10-50 \%)$. A similar trend was seen for chymotrypsinlike substrate using metallo, trypsin-like and elastase-like protease inhibitors $(\sim 10-40 \%)$.

Conclusion This investigation has suggested that there is significant non-specific hydrolysis and cross-reactivity when fluorogenic substrates are utilised to measure active proteases in complex biological samples. Thus, using such fluorogenic substrates may produce elevated readings, impacting on the accuracy of results when such assays are used for clinical or research purposes.

\section{S135 CIRCULATING METABOLITES IN CHRONIC THROMBOEMBOLIC PULMONARY HYPERTENSION AND CHRONIC THROMBOEMBOLIC PULMONARY VASCULAR OCCLUSION}

${ }^{1} \mathrm{KI}$ Zalewska, ${ }^{1} \mathrm{EM}$ Swietlik, 'J Sanchez Hernandez, ${ }^{1} \mathrm{JE}$ Cannon, ${ }^{1} \mathrm{D}$ Taboada, ${ }^{2} \mathrm{M}$ Newnham, ${ }^{2} \mathrm{C}$ Hadinnapola, ${ }^{2} \mathrm{NW}$ Morrell, ${ }^{1} \mathrm{MR}$ Toshner, IJ Pepke Zaba. ${ }^{1}$ Papworth Hospital NHS Foundation Trust, Cambridge, UK; ${ }^{2}$ University of Cambridge, Cambridge, UK

\subsection{6/thoraxjnl-2016-209333.141}

Introduction Recent studies have demonstrated that metabolomic profiling can identify metabolites and pathways which may have importance in the pathobiology of pulmonary arterial hypertension. However, the plasma metabolome in chronic thromboembolic pulmonary hypertension (CTEPH) and chronic thromboembolic vascular occlusions without pulmonary hypertension (CTED) has not been well characterised.

Objective To profile circulating metabolites in CTEPH and CTED and assess metabolite gradients across the pulmonary circulation.

Methods In the patient group, multisite blood sampling was performed at the time of right heart catheterisation. Blood samples were collected from the superior vena cava, pulmonary artery and radial artery.

Venous blood samples from patients were compared to healthy controls to identify the metabolites present and to assess the difference between health and disease. Additionally, in the disease group, transpulmonary gradients were assessed by analysis of fold change in metabolite concentration between paired samples from the pulmonary artery and radial artery.

Untargeted, semi-quantitative metabolic profiling of plasma was performed using the Metabolon DiscoveryHD4 ${ }^{\mathrm{TM}}$ platform (Metabolon, NC, USA), utilising 2 ultra-high performance liquid chromatography methods, coupled with tandem mass spectrometry. Kruskal-Wallis analysis was used to compare metabolites between disease and control, with false discovery rate correction for multiple testing.

Results The disease group included patients with a spectrum of chronic pulmonary vascular occlusions (Table 1). A total of 1375 metabolites were detected in 70 venous plasma samples analysed from 43 patients and 27 healthy controls. Amongst endogenous metabolites, 266 showed a significant difference between disease and control. In the disease group there were increases in acylcarnitine metabolites, long chain fatty acids, polyamines, glycogen metabolites and primary bile acid metabolites compared to healthy controls. There was a reduction in lysolipids, plasmalogens, aminosugars, branched chain amino acid metabolites, glutathione metabolites and a number of steroids (Table 1). Analysis of transpulmonary gradients revealed primarily a reduction in metabolite concentration across the pulmonary circulation. This included depletion of energy substrates, lysolipids, lysoplasmalogens and acylcholines.

Conclusions This pilot study of circulating metabolites in patients with CTEPH, CTED and healthy controls reveals differences between health and disease in several biological pathways. Measurement of the transpulmonary gradient of metabolites indicated predominant clearance of circulating metabolites associated with energy metabolism and cell turnover. These findings require confirmation in a larger population.

Abstract S135 Table 1 Study population and changes in metabolite groups in venous blood of patients compared to healthy controls

\begin{tabular}{|c|c|c|}
\hline & $\begin{array}{l}\text { Chronic pulmonary vascular } \\
\text { occlusions ( } n=43 \text { ) }\end{array}$ & $\begin{array}{l}\text { Controls } \\
(\mathrm{n}=27)\end{array}$ \\
\hline \multicolumn{3}{|l|}{ Group demographics } \\
\hline Age (years) & $58(22-77)$ & $\begin{array}{l}44(18- \\
75)\end{array}$ \\
\hline Sex (\% male) & 64 & 60 \\
\hline \multicolumn{3}{|l|}{ Patient group } \\
\hline Proximal CTEPH- treatment naive ( $\mathrm{n}$ ) & 11 & \\
\hline Distal CTEPH (n) & 3 & \\
\hline $\begin{array}{l}\text { Proximal CTEPH- previous pulmonary } \\
\text { endarterectomy- residual PH ( } \mathrm{n} \text { ) }\end{array}$ & 11 & \\
\hline $\begin{array}{l}\text { Proximal CTEPH- previous pulmonary } \\
\text { endarterectomy, no residual PH ( } \mathrm{n} \text { ) }\end{array}$ & 10 & \\
\hline $\begin{array}{l}\text { Chronic thromboembolic vascular occlusions } \\
\text { without } \mathrm{PH}(\mathrm{n} \text { ) }\end{array}$ & 8 & \\
\hline \multicolumn{3}{|l|}{ Changes in metabolites in disease } \\
\hline Acylcarnitines & $\uparrow$ & \\
\hline Long chain fatty acids & $\uparrow$ & \\
\hline Polyamines & $\uparrow$ & \\
\hline Glycogen metabolites & $\uparrow$ & \\
\hline Primary bile acid metabolites & $\uparrow$ & \\
\hline Lysolipids & $\downarrow$ & \\
\hline Plasmalogens & $\downarrow$ & \\
\hline Aminosugars & $\downarrow$ & \\
\hline Branched chain amino acids & $\downarrow$ & \\
\hline Glutathione metabolites & $\downarrow$ & \\
\hline Steroids & $\downarrow$ & \\
\hline
\end{tabular}

\section{S136 POTENTIAL THERAPEUTIC BENEFITS OF THE HUMAN AMNIOTIC EPITHELIUM CELL SECRETOME DURING EX- VIVO PERFUSION OF DONOR LUNGS}

${ }^{1} J W$ Mayes, ${ }^{2} \mathrm{~K}$ Jiwa, ${ }^{3} \mathrm{~B}$ Leaw, ${ }^{3} \mathrm{~J}$ Tan, ${ }^{3} \mathrm{~S}$ Lau, 'L Borthwick, 'A Andreasson, 'J Dark, ${ }^{3} \mathrm{G}$ Jenkin, ${ }^{3} \mathrm{R}$ Lim, ${ }^{1} \mathrm{AJ}$ Fisher. ${ }^{1}$ Newcastle University, Newcastle upon Tyne, UK; ${ }^{2}$ Freeman Hospital Newcastle upon Tyne NHS Hospitals, Newcastle upon Tyne, UK; ${ }^{3}$ The Ritchie Centre Monash University, Melbourne, Australia

\subsection{6/thoraxjnl-2016-209333.142}

Introduction Ex-vivo lung perfusion (EVLP) is used to assess and potentially recondition donor lungs that are not initially suitable for transplantation. In a recent UK study, EVLP was associated 